\title{
Observations of the bright radio sources in the North Celestial Pole region at the RATAN-600 radio telescope
}

\author{
M. G. Mingaliev ${ }^{1}$, V. A. Stolyarov ${ }^{1,2}$, R. D. Davies ${ }^{3}$, S. J. Melhuish ${ }^{4}$, N. A. Bursov ${ }^{1}$, and G. V. Zhekanis ${ }^{1}$ \\ 1 Special Astrophysical Observatory of Russian Academy of Sciences, Nizhnij Arkhyz, \\ Karachaevo-Cherkessia Republic 357147, Russia \\ e-mail: marat@sao.ru; vlad@sao.ru; nnb@ratan.sao.ru; gvz@ratan.sao.ru \\ 2 University of Cambridge, Institute of Astronomy, Madingley Rd., Cambridge CB3 OHA, UK \\ e-mail: vlad@ast.cam.ac.uk \\ 3 University of Manchester, Jodrell Bank Observatory, Macclesfield, Cheshire SK11 9DL, UK \\ e-mail: rdd@jb.man.ac.uk \\ 4 University of Wales, Cardiff, Department of Physics and Astronomy, 5, The Parade, Cardiff CF24 3YB, UK \\ e-mail: Simon.Melhuish@astro.cf.ac.uk
}

Received 3 November 2000 / Accepted 2 February 2001

\begin{abstract}
A survey of the North Celestial Pole region using the RATAN-600 radio telescope at five frequencies in the range 2.3 to $21.7 \mathrm{GHz}$ is described. Sources were chosen from the NVSS catalogue. The flux densities of 171 sources in the Declination range $+75^{\circ}$ to $+88^{\circ}$ are presented; typical flux density errors are $5-10$ percent including calibration errors. About 20 percent of the sources have flat spectra or a flat component.
\end{abstract}

Key words. radio astronomy - radio continuum - galaxies

\section{Introduction}

In the current paper we present the results of observations of bright radio sources in the North Celestial Pole (NCP) region within the declination range of $+75^{\circ} \leq \delta \leq$ $+88^{\circ}$ taken with the RATAN-600 radio telescope of the Russian Academy of Sciences (Korolkov \& Pariiskii 1979; Parijskij 1993). This NCP survey was initiated as a compliment to the $5 \mathrm{GHz}$ interferometric study of Galactic foreground emission in the NCP made at Jodrell Bank in 1998-1999 (Melhuish et al. 2001). In order to obtain information about Galactic synchrotron and free-free emission in the survey area it was necessary to determine the $5 \mathrm{GHz}$ flux densities of the point sources in the area and remove their contribution from the map.

Up to the present time, there has been no sensitive survey of the NCP region at frequencies higher than the 1.4 GHz NRAO VLA Sky Survey (NVSS), (Condon et al. 1998). The 5 GHz Greenbank survey (Gregory et al. 1996) only extends northwards as far as $\delta=+75^{\circ}$. There is limited data available at $5 \mathrm{GHz}$ from the early survey at $+88^{\circ} \leq \delta \leq+90^{\circ}$ of Pauliny-Toth et al. (1978) and

Send offprint requests to: V. Stolyarov,

e-mail: vlad@ast.cam.ac.uk from the Kuehr et al. (1981) catalogue of bright sources. Furthermore, since a sizable fraction (perhaps as many as $20 \%$ ) of $5 \mathrm{GHz}$ sources may have flat spectra and are variable, a contemporary survey covering $5 \mathrm{GHz}$ was required for the NCP project. The upper Declination limit of the present RATAN-600 survey was set at $+88^{\circ}$ since the telescope is used in transit mode and data cannot be collected close to the NCP in this mode.

\section{Selection criteria for the survey}

The aim of this NCP survey was to obtain information about bright point sources which might make a significant contribution to the $5 \mathrm{GHz}$ degree-scale interferometer survey of foreground Galactic emission in the NCP (Melhuish et al. 2001). The interferometer has a resolution of $2^{\circ}$ and a temperature/flux density sensitivity of $60 \mu \mathrm{K}$ in antenna temperature per Jansky. In order to achieve a survey sensitivity approaching $10 \mu \mathrm{K}$ it was decided to measure directly with RATAN-600 all those sources giving $10 \mu \mathrm{K}$ or more with the interferometer, corresponding to a flux density $S \geq 150 \mathrm{mJy}$. At this flux density there is one source per interferometer beam area of $2^{\circ} \times 2^{\circ}$. 
Table 1. Parameters of the receivers used in the survey. See text for meaning of the symbols

\begin{tabular}{c|c|c|c|c|c}
\hline$\nu_{\mathrm{c}}, \mathrm{GHz}$ & $\Delta \nu, \mathrm{GHz}$ & $\Delta T, \mathrm{mK}$ & $T_{\mathrm{phys}}, \mathrm{K}$ & $T_{\mathrm{ampl}}, \mathrm{K}$ & $T_{\mathrm{sys}}, \mathrm{K}$ \\
\hline 21.7 & 2.5 & 3.5 & 15 & 23 & 77 \\
11.2 & 1.4 & 3.0 & 15 & 18 & 70 \\
7.7 & 1.0 & 3.0 & 15 & 14 & 62 \\
3.9 & 0.6 & 2.5 & 15 & 8 & 37 \\
2.3 & 0.4 & 8.0 & 310 & 35 & 95 \\
\hline
\end{tabular}

The sources chosen for measurement were taken from the $1.4 \mathrm{GHz}$ NVSS catalogue, the catalogue covering the $\mathrm{NCP}$ region which is nearest in frequency to $5 \mathrm{GHz}$. A 150 mJy flux density limit at $5 \mathrm{GHz}$ corresponds to 350-400 mJy at $1.4 \mathrm{GHz}$ assuming an average spectral index of $0.7\left(S \propto \nu^{-\alpha}\right)$ for the sources. Accordingly, the adopted "complete sample" criteria for the sources selected from the NVSS catalogue were:

1. Flux density $S_{\nu} \geq 400 \mathrm{mJy}$ at the NVSS frequency of $1.4 \mathrm{GHz}$;

2. $00^{\mathrm{h}} \leq \alpha \leq 24^{\mathrm{h}}$

3. $+75^{\circ} \leq \delta \leq+88^{\circ}$.

In total we have selected for observation 182 objects which satisfy these criteria.

\section{The observations}

The observations were made in February-March 1999 using the South sector of the RATAN-600 reflector - type radio telescope at 2.3, 3.9, 7.7, 11.2 and 21.7 GHz (Parijskij 1993; Berlin et al. 1997; Berlin \& Friedman 1996). The parameters of the receivers are listed in Table 1 , where $\nu_{\mathrm{c}}$ is the central frequency, $\Delta \nu$ is the bandwidth, $\Delta T$ is the sensitivity of the radiometer over $1 \mathrm{~s}$ integration, $T_{\text {phys }}$ is the physical temperature of the radiometer amplifier, $T_{\mathrm{ampl}}$ is the noise temperature of the amplifier and $T_{\text {sys }}$ is the noise temperature of the whole system at the given frequency. All of the radiometers have HEMT first-stage amplifiers.

Information about $F W H M$ can be found in the article by Kovalev et al. (1999). For example at $11.2 \mathrm{GHz}$ the $F W H M$ is about $17^{\prime \prime} \times 2^{\prime}$ at the elevations of the NCP observations.

Usually each source was observed 5-8 times per set. Scans of all of the sources were corrected for baseline slope when fitted to a Gaussian response using data reduction software developed by Verkhodanov (1997). The accuracy of the antenna temperature of each source was determined as the standard error of the mean from the $N$ observations of the set.

\section{Calibration and data reduction}

The calibration of our observations is a challenging task. There are no radio astronomical calibrators listed in this area of the sky. The only place where we have some information about source fluxes in a wide frequency range
$(0.325-42 \mathrm{GHz})$ in the NCP region is the VLA Calibrator List (Perley \& Taylor 1999). However, the fluxes listed there are approximate because most of the sources from the VLA List are compact and, hence, variable. To address this problem we selected for our purpose only sources with steep spectra that are not likely to be variable and, if possible, with minimal expected VLA amplitude closure errors (about $3 \%$ ). The fluxes of the calibrators from the VLA Calibrator List are listed at 90, 20, 6, 3.7, 2 and $0.7 \mathrm{~cm}$ wavelength bands $(0.325,1.5,5,8.1,15$ and $42.9 \mathrm{GHz}$ respectively). In order to get fluxes at the RATAN-600 frequency bands the spectra of the calibrators were interpolated to the desired frequencies by second order polynomial.

\subsection{The calibration errors, $\sigma_{c}$}

The flux density measurement procedure at the RATAN600 is described by Aliakberov et al. (1985). The response of the antenna to a source with known flux density at a given frequency $\nu$ is a function of antenna elevation (Mingaliev et al. 1998), which may be expressed as:

$T_{\text {ant }, \nu}=F_{\nu}\left(S_{\nu}, e\right)=S_{\nu} f_{\nu}(e) \quad S_{\nu}=T_{\text {ant }, \nu} g_{\nu}(e)$,

where

$g_{\nu}(e)=1 / f_{\nu}(e)$ - elevation calibration function;

$T_{\text {ant }, \nu} \quad$ - antenna temperature;

$F_{\nu}, f_{\nu} \quad$ - arbitrary functions;

$e=90-\phi+\delta$ - antenna elevation;

$\phi=43.65333 \quad$ - latitude of the telescope site.

In order to get the flux density from $T_{\text {ant }, \nu}$ we have to multiply it by the elevation calibration function $g_{\nu}(e)$, which is believed to be a second order polynomial (Trushkin 1985). To get an estimate of this function we observe the calibration sources of known flux density spanning a wide range of declination, $\delta$. Having the list of values

$g_{\nu}\left(e_{i}\right)=S_{\nu, i} / T_{\mathrm{ant}, \nu, i}$

for different sources we can approximate the functions $g_{\nu}(e)$ by a second order polynomial with the help of minimization of the mean square value of the estimated error (least square estimator).

The names of calibration sources we used and their adopted flux densities are listed in the Table 2. The assumed flux density errors are $3 \%$ as given in the VLA Calibrator List.

The calibration curves $g_{\nu}(e)$ for $2.3,3.9,7.7$ and 11.2 GHz are given in Fig. 1. A second order polynomial fit was made to the observational data at each frequency. We found the errors in the calibration curves were 11 , $10.3,2.4,5.6$ and $7.4 \%$ at $21.7,11.2,7.7,3.9$ and $2.3 \mathrm{GHz}$ respectively. The total calibration error is the quadratic addition of the $3 \%$ VLA Calibration List error and the error from the $g_{\nu}(e)$ calibration curve. 


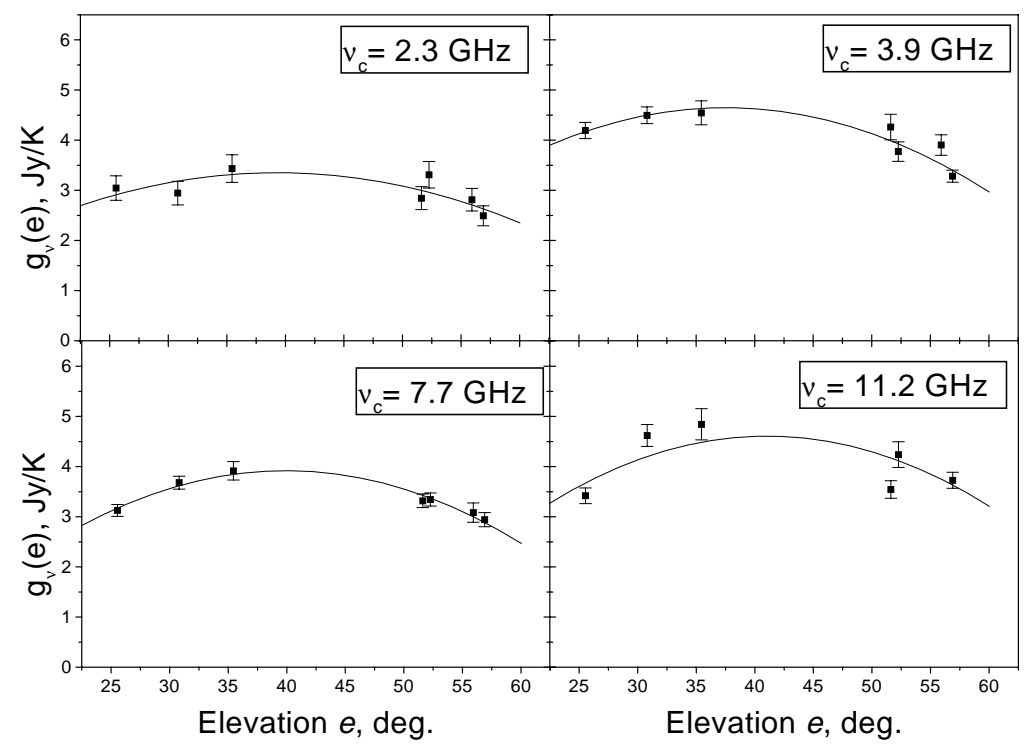

Fig. 1. Calibration curves for $2.3,3.9,7.7$ and $11.2 \mathrm{GHz}$ bands

Table 2. Adopted calibrator source flux densities. Sources are from the VSA Calibrator List; errors are assumed to be $3 \%$

\begin{tabular}{l|c|c|c|c|c}
\hline $\begin{array}{l}\text { Name } \\
\text { of the source }\end{array}$ & $\begin{array}{c}S\left(\nu_{\mathrm{c}}=21.7 \mathrm{GHz}\right), \\
\mathrm{Jy}\end{array}$ & $\begin{array}{c}S\left(\nu_{\mathrm{c}}=11.2 \mathrm{GHz}\right), \\
\mathrm{Jy}\end{array}$ & $\begin{array}{c}S\left(\nu_{\mathrm{c}}=7.7 \mathrm{GHz}\right), \\
\mathrm{Jy}\end{array}$ & $\begin{array}{c}S\left(\nu_{\mathrm{c}}=3.9 \mathrm{GHz}\right), \\
\mathrm{Jy}\end{array}$ & $\begin{array}{c}S\left(\nu_{\mathrm{c}}=2.3 \mathrm{GHz}\right), \\
\mathrm{Jy}\end{array}$ \\
\hline J0017+815 & 0.48 & 0.78 & 0.94 & 1.00 & 0.9 \\
J0229+777 & - & - & 0.41 & 1.05 & 1.8 \\
J0410+769 & 1.14 & 1.77 & 2.23 & 3.35 & 4.49 \\
J0626+820 & 0.31 & 0.56 & 0.74 & 0.74 & 0.95 \\
J1435+760 & - & 0.31 & 2.27 & 3.69 & 5.03 \\
J1459+716 & 1.00 & 1.72 & 0.43 & 0.44 & 0.46 \\
J2022+761 & 0.42 & 0.41 & &
\end{tabular}

\subsection{The errors of $T_{\text {ant }}$ measurements, $\sigma_{m}$}

The specifics of the RATAN-600 observations lead to the fact that the errors of the antenna temperature measurements depend not only on the receiver noise, but also on the atmospheric fluctuations on the scale of the main beam, on the accuracy of antenna surface setting for the actual source observation and on the accuracy of the feed cabin positioning (the cabin with secondary mirror and receivers).

Generally speaking, the part of these errors due to the receiver noise can be estimated according to the formula

$\Delta T_{\mathrm{rec}}=\Delta T /(\Delta t N k)^{1 / 2}$

where $\Delta T$ is the sensitivity of the receiver over $1 \mathrm{~s}$ (listed in Table 1); $\Delta t$ is an integration time, the time that the source takes to cross the main beam of the antenna during the drift scan; $N$ is the number of the drift scans; $k$ is equal to 1 for single horn receivers $(2.3$ and $3.9 \mathrm{GHz})$ and 2 for beam-switching receivers $(7.7,11.2,21.7 \mathrm{GHz})$, where we can take into account both positive and negative beams. The variable parameter is $\Delta t$, because it depends on the width of the main beam which is different for different frequencies, and varies with declination. In the case of the NCP declination range and the frequency range 21.7 to $2.3 \mathrm{GHz}, \Delta t$ lies in the range 1-15 s. As an example for
$3.9 \mathrm{GHz}, \delta=+75^{\circ}, \Delta t=4 \mathrm{~s}$ and $N=5, \Delta T_{\text {rec }}$ is equal to $0.56 \mathrm{mK}$.

Unfortunately the contribution of atmospheric fluctuations increases as $\Delta t$ increases corresponding to larger angular scales thereby partially reducing the growth of sensitivity expected from longer integration times. The errors related to the accuracy of antenna surface setting and feed cabin positioning are more complicated to account for. The feed cabin position errors are most important for high frequency observations; as an example it is necessary to position the cabin with an accuracy of $0.1 \lambda$, which is $1.4 \mathrm{~mm}$ in the case of $21.7 \mathrm{GHz}$.

However, estimating of the antenna temperature of the source for every drift scan (e.g by Gaussian fitting) and then calculating the variance of the $T_{\text {ant }}$ for the $N$ observations of the data set can give us the measurement error, $\sigma_{\mathrm{m}}$, including all of the components listed above.

\subsection{The total errors, $\sigma_{t}$}

The total fractional error in the flux densities listed in this survey is the quadratic sum of the total calibration error 
and the error in the antenna temperature measurement, namely,

$$
\left(\frac{\sigma_{\mathrm{t}}}{S_{\nu}}\right)^{2}=\left(\frac{\sigma_{\mathrm{c}}}{g_{\nu}(e)}\right)^{2}+\left(\frac{\sigma_{\mathrm{m}}}{T_{\mathrm{ant}, \nu}}\right)^{2}
$$

where

$$
\begin{array}{ll}
\sigma_{\mathrm{t}} & \text { - total standard error; } \\
\sigma_{\mathrm{c}} & \text { - standard error of calibration; } \\
\sigma_{\mathrm{m}} & \text { - standard error of } T_{\mathrm{ant}, \nu} \text { measurement; } \\
S_{\nu} & \text { - flux density; } \\
g_{\nu}(e)=1 / f_{\nu}(e) & \text { - elevation calibration function; } \\
T_{\mathrm{ant}, \nu} & \text { - antenna temperature. }
\end{array}
$$

The values of the standard error of $T_{\mathrm{ant}, \nu}$ measurement, $\sigma_{\mathrm{m}}$, are $2-3 \%$ for $11.2,7.7$ and $3.9 \mathrm{GHz}, 3-5 \%$ for $2.3 \mathrm{GHz}$ and $7-11 \%$ for $21.7 \mathrm{GHz}$. For the brighter sources $\sigma_{\mathrm{m}}$ is typically half these values, indicating highly consistent observations. Thus at the frequencies of 21.7, 11.2 and $3.9 \mathrm{GHz}$ the calibration errors dominate.

\subsection{Comparison with the other catalogues}

The RATAN-600 results described in this paper are in good accordance with the flux densities given by NVSS at 1.4 GHz, the Westerbork Northern Sky Survey (WENSS; Rengelink et al. 1997) at $0.325 \mathrm{GHz}$ and the earlier data of the Kuehr (1981) Catalogue. Four sample spectra are shown in Fig. 2 which compare the RATAN-600 data with those from the three above catalogues. The sources illustrated all have steep spectra and as a consequence are not likely to be variable. The few discrepancies in the plotted spectra are all in the older Kuehr data.

\section{Results}

The spectra for 171 sources in the present RATAN-600 NCP survey are given in Table 4. Data from WENSS and NVSS are included. Nearly all the sources have complete data at 2.3, 3.9, 7.7 and $11.2 \mathrm{GHz}$; 40 sources have 21.7 GHz flux densities. Only a few of these sources have been observed previously over this wide frequency range. The columns in the table are:

Column 1: $\quad$ The source name (NVSS notation), corresponding to epoch J2000 coordinates;

Columns 2-3: The flux density in Jy and standard error at $0.325 \mathrm{GHz}$ (WENSS catalogue, Rengelink et al. 1997);

Columns 4-5: The flux density in Jy and standard error at $1.4 \mathrm{GHz}$ (NVSS catalogue, Condon et al. 1998);

Columns 6-15: The flux density in Jy and total standard error, $\sigma_{\mathrm{t}}$, at $2.3,3.9,7.6,11.2$ and $21.7 \mathrm{GHz}$ respectively;

Column 16: The spectral index $\alpha=-\log \left(S\left(\nu_{1}\right) /\right.$ $\left.S\left(\nu_{2}\right)\right) / \log \left(\nu_{1} / \nu_{2}\right)$, computed between fluxes at 0.325 and $11.2 \mathrm{GHz}$ (or the nearest available frequencies).
A number of the sources in the NVSS target list were not fully resolved in the RATAN-600 observations, largely as a result of the more extended beam in the declination direction. These closely adjacent sources are listed as a single entry in Table 4 and are designated as RAXXX and DecXXX. The listed flux densities of these complexes are the sum of the flux densities of the individual sources. The NVSS sources contributing to each of the 7 complexes are given in Table 3 .

\section{Discussion}

Some preliminary comments are worthwhile on the multifrequency data for this NCP survey in which 171 individual sources were identified.

\subsection{The contribution to $5 \mathrm{GHz}$ interferometry}

The first aim of these observations was to obtain a list of those sources which would contribute at a significant level to our $5 \mathrm{GHz}$ survey (Melhuish et al. 2001) of the NCP. The chosen limit to the flux density at $5 \mathrm{GHz}$ was $150 \mathrm{mJy}$ which corresponds to a signal amplitude of $10 \mu \mathrm{K}$ in the interferometer. The majority of sources (80 percent) were stronger than this limit and would make a significant contribution to the CMB foreground and should be removed from the interferometer survey.

The question then arises as to the further contribution from flat spectrum and rising spectrum sources not included in our survey which would have a $5 \mathrm{GHz}$ flux density of $\geq 150 \mathrm{mJy}$. Remembering that our source selection criterion was 400 mJy at $1.4 \mathrm{GHz}$, a spectral index of 0.7 gives a flux density of $150 \mathrm{mJy}$ at $5 \mathrm{GHz}$. A source spectral index of 0.2 will give twice this limit; only 10 percent of our sources chosen at $1.4 \mathrm{GHz}$ have spectral indices flatter than this value. Accordingly there will be a further contribution from such sources with flux densities at $1.4 \mathrm{GHz}$ of 200-400 mJy. Assuming the fraction of flat spectrum sources stays constant with decreasing frequency, we may expect $\sim 5$ sources in this category. Yet another contribution will come from Gigahertz Peaked Spectrum (GPS) sources; likewise there will be $\sim 5$ extra sources with a flux density above $150 \mathrm{mJy}$ at $5 \mathrm{GHz}$.

\subsection{Statistics of sources spectra}

Although this is a modest sample of $\mathrm{GHz}$ spectra, it provides an indication of the spectral properties of the brightest radio sources in the NCP region $\left(+75^{\circ} \leq \delta \leq\right.$ $\left.+88^{\circ}\right)$. We would expect them to follow the trends in the general field. One particular advantage of the present catalogue is that all the sources were observed simultaneously at all frequencies to provide an instantaneous spectrum unaffected by source variability. The histograms of spectral index values estimated over the frequency ranges $0.325 / 1.4 \mathrm{GHz}, 0.325 / 3.9 \mathrm{GHz}, 3.9 / 11.2 \mathrm{GHz}$ and $0.325 / 11.2 \mathrm{GHz}$ are presented in Figs. 3-6 respectively. 


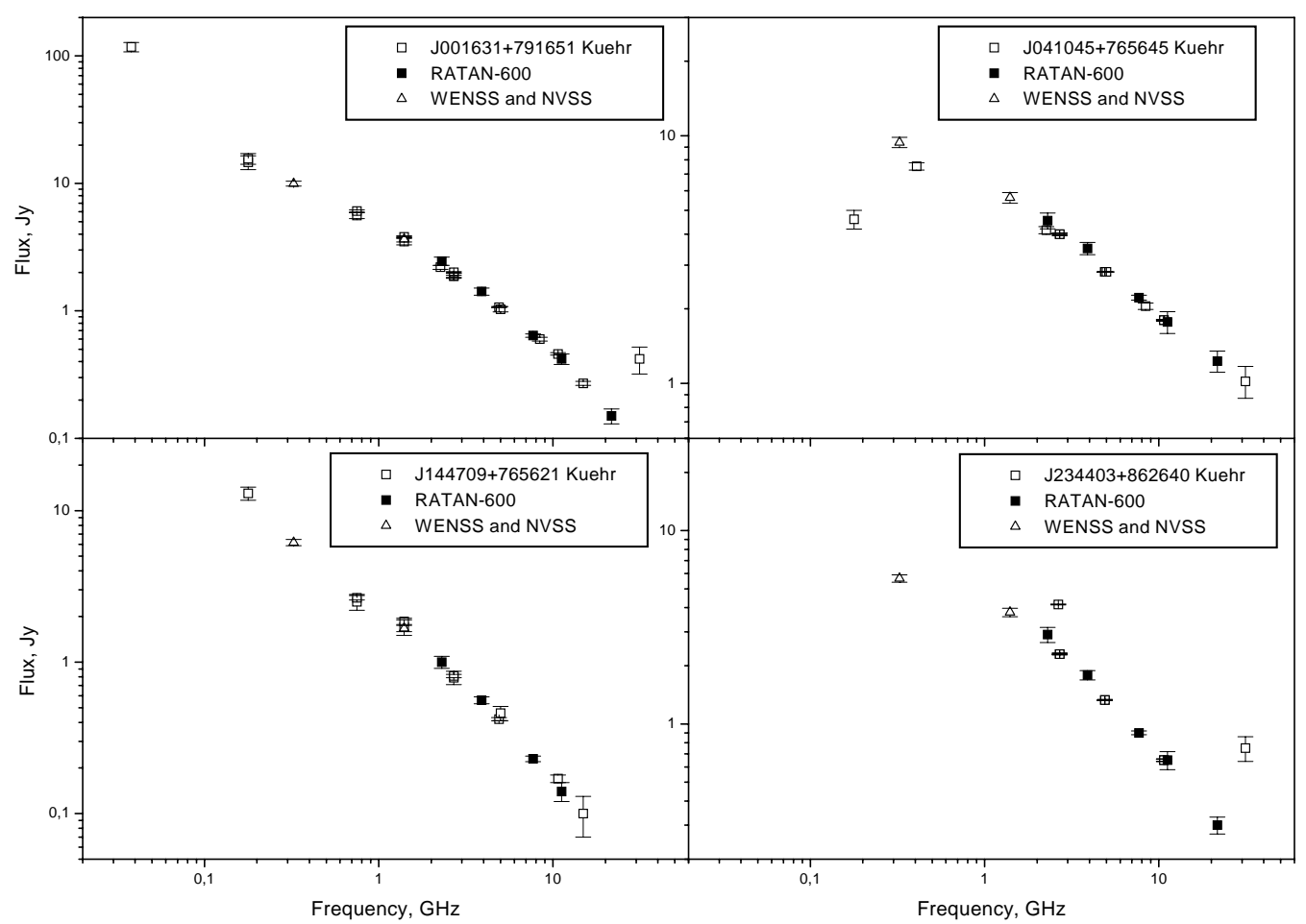

Fig. 2. Four sample source spectra from Kuehr catalogue compared with RATAN-600, NVSS and WENSS data

Table 3. Complex sources

\begin{tabular}{|c|c|c|c|}
\hline Name in NVSS & Name in the Table 4 & Name in NVSS & Name in the Table 4 \\
\hline $\bar{J} 022235+861727$ & \multirow[t]{4}{*}{ J0222XX+861XXX } & $\mathrm{J} 184142+794752$ & \multirow[t]{4}{*}{ J184XXX+794XXX } \\
\hline $\mathrm{J} 022248+861851$ & & $\mathrm{~J} 184151+794727$ & \\
\hline J022249+862027 & & $\mathrm{J} 184214+794613$ & \\
\hline & & $\mathrm{J} 184226+794517$ & \\
\hline J074246+802741 & \multirow[t]{3}{*}{ J074XXX+802XXX } & & \multirow{3}{*}{$\mathrm{J} 20425 \mathrm{X}+750 \mathrm{XXX}$} \\
\hline $\mathrm{J} 074305+802544$ & & $\mathrm{~J} 204257+750428$ & \\
\hline & & $\mathrm{J} 204259+750306$ & \\
\hline $\mathrm{J} 101330+855411$ & \multirow[t]{5}{*}{ J101XXX+855XXX } & & \multirow{3}{*}{ J2118XX+751XXX } \\
\hline \multirow[t]{4}{*}{$\mathrm{J} 101412+855349$} & & $\mathrm{~J} 211814+751203$ & \\
\hline & & $\mathrm{J} 211817+751112$ & \\
\hline & & $\mathrm{J} 235521+795552$ & \multirow[t]{2}{*}{$\mathrm{J} 2355 \mathrm{XX}+795 \mathrm{XXX}$} \\
\hline & & $\mathrm{J} 235525+795442$ & \\
\hline
\end{tabular}

The majority of the sources have spectral indices in the range 0.6 to 1.5 at $\mathrm{GHz}$ frequencies. The canonical steepening of synchrotron spectra at higher frequencies is evident in the data. The median spectral index in the range $0.325 / 1.4 \mathrm{GHz}$ is 0.78 . This value rises to 0.82 for $1.4 / 2.3 \mathrm{GHz}$, to 0.95 for $2.3 / 3.9 \mathrm{GHz}$ and to 1.15 for $3.9 / 11.2 \mathrm{GHz}$. At the higher part of this frequency range there is an increasing spread in the range of spectral indices which indicates that the turn-over of the spectrum occurs at a range of $\mathrm{GHz}$ frequencies. This broadening of the histogram is readily seen in Fig. 5 where a significant number ( $\sim 30$ percent) have a spectral index greater than 1.2 in the frequency range $3.9 / 11.2 \mathrm{GHz}$; a small fraction ( 10 percent) of these higher spectral indices are a result of the significant total error, $\sigma_{\mathrm{t}}$, on weaker sources at $11.2 \mathrm{GHz}$.
The fraction of flatter spectrum sources in our $\mathrm{GHz}$ NCP survey, as illustrated in Figs. 4-6, is 20-25 percent. This family of flattish spectrum sources is of particular concern as a foreground in the measurement of fluctuations in the cosmic microwave background.

\subsection{Individual sources with compact components}

The spectral signature of compact radio sources is a flat component arising from synchrotron self-absorption. Such a component may be seen as a flat spectrum over a wide frequency range, a flat spectrum component at a high frequency emerging from a steep spectrum low-frequency source or a GHz Peaked Spectrum source. Such spectra are found in some 20 percent of the 171 sources of the present survey. 14 sources show a relatively flat spectrum over the 
Table 4. Fluxes of the sources in the NCP region

\begin{tabular}{|c|c|c|c|c|c|c|c|c|c|c|c|c|c|c|c|}
\hline $\begin{array}{l}\text { Name, } \\
\text { NVSS }\end{array}$ & $\begin{array}{r}S_{0.325} \\
\mathrm{Jy}, \\
\end{array}$ & $\begin{array}{r}\sigma_{\mathrm{t}} \\
\text { WENSS } \\
\end{array}$ & $\begin{array}{r}S_{1.4} \\
\mathrm{Jy}, \\
\end{array}$ & $\begin{array}{r}\sigma_{\mathrm{t}} \\
\text { NVSS } \\
\end{array}$ & $\begin{array}{r}S_{2.3} \\
\mathrm{Jy} \\
\end{array}$ & $\overline{\sigma_{\mathrm{t}}}$ & $\begin{array}{r}S_{3.9} \\
\mathrm{Jy} \\
\end{array}$ & $\sigma_{\mathrm{t}}$ & $\begin{array}{r}S_{7.7} \\
\mathrm{Jy} \\
\end{array}$ & $\sigma_{\mathrm{t}}$ & $\begin{array}{r}S_{11.2} \\
\mathrm{Jy} \\
\end{array}$ & $\sigma_{\mathrm{t}}$ & $\begin{array}{r}S_{21.7} \\
\mathrm{Jy} \\
\end{array}$ & $\sigma_{\mathrm{t}}$ & $\begin{array}{r}\text { Sp. index } \\
\alpha_{0.325 / 11.2} \\
\end{array}$ \\
\hline J000943+772440 & 2.059 & 0.082 & 0.628 & 0.021 & 0.447 & 0.04 & 0.267 & 0.017 & 0.161 & 0.007 & 0.14 & 0.016 & - & - & 0.76 \\
\hline J001236+854313 & 1.605 & 0.064 & 0.691 & 0.023 & 0.539 & 0.048 & 0.246 & 0.018 & 0.148 & 0.005 & 0.099 & 0.011 & - & - & 0.79 \\
\hline J001311+774846 & 4.517 & 0.181 & 2.101 & 0.071 & 1.578 & 0.141 & 0.981 & 0.056 & 0.467 & 0.012 & 0.314 & 0.034 & 0.202 & 0.03 & 0.75 \\
\hline J001631+791651 & 9.959 & 0.398 & 3.651 & 0.123 & 2.46 & 0.183 & 1.422 & 0.087 & 0.643 & 0.017 & 0.422 & 0.044 & 0.145 & 0.022 & 0.89 \\
\hline J001708+813508 & 0.688 & 0.028 & 0.693 & 0.023 & 0.853 & 0.067 & 1.022 & 0.058 & 0.919 & 0.023 & 0.788 & 0.082 & 0.441 & 0.065 & -0.04 \\
\hline J001816+782743 & 2.16 & 0.086 & 0.707 & 0.024 & 0.428 & 0.038 & 0.252 & 0.015 & 0.109 & 0.005 & 0.054 & 0.006 & - & - & 1.04 \\
\hline J003812+844727 & 0.972 & 0.039 & 0.406 & 0.014 & 0.332 & 0.03 & 0.223 & 0.017 & 0.108 & 0.005 & 0.084 & 0.01 & - & - & 0.69 \\
\hline J011045+873822 & 2.215 & 0.089 & 0.686 & 0.023 & 0.467 & 0.042 & 0.206 & 0.015 & 0.118 & 0.005 & 0.081 & 0.009 & - & - & 0.93 \\
\hline J013156+844612 & 2.072 & 0.083 & 0.777 & 0.026 & 0.603 & 0.054 & 0.361 & 0.027 & 0.186 & 0.005 & 0.136 & 0.016 & - & - & 0.77 \\
\hline J015207+755035 & 2.823 & 0.113 & 0.839 & 0.028 & 0.526 & 0.047 & 0.285 & 0.016 & 0.095 & 0.005 & 0.054 & 0.006 & - & - & 1.12 \\
\hline J020537+752207 & 0.86 & 0.035 & 1.151 & 0.039 & 0.873 & 0.078 & 0.611 & 0.036 & 0.309 & 0.009 & 0.202 & 0.021 & 0.097 & 0.015 & 0.41 \\
\hline J020723+795602 & 3.89 & 0.156 & 1.363 & 0.046 & 0.932 & 0.072 & 0.582 & 0.033 & 0.252 & 0.008 & 0.18 & 0.021 & - & - & 0.87 \\
\hline $\mathrm{J} 0222 \mathrm{XX}+861 \mathrm{XXX}$ & 20.538 & 0.822 & 6.478 & 0.217 & 4.006 & 0.422 & 3.006 & 0.261 & 1.137 & 0.133 & 0.813 & 0.095 & - & - & 0.91 \\
\hline J022454+765554 & 5.949 & 0.238 & 1.927 & 0.065 & 1.271 & 0.095 & 0.744 & 0.042 & 0.334 & 0.009 & 0.21 & 0.023 & 0.085 & 0.013 & 0.94 \\
\hline J022914+774316 & 8.067 & 0.323 & 2.683 & 0.09 & 1.663 & 0.124 & 0.95 & 0.054 & 0.401 & 0.011 & 0.259 & 0.028 & 0.111 & 0.016 & 0.97 \\
\hline J023010+814129 & 2.285 & 0.091 & 1.048 & 0.035 & 0.783 & 0.07 & 0.544 & 0.031 & 0.284 & 0.008 & 0.213 & 0.023 & 0.212 & 0.031 & 0.67 \\
\hline J025417+791147 & 2.186 & 0.088 & 0.643 & 0.022 & 0.463 & 0.041 & 0.212 & 0.013 & 0.105 & 0.006 & 0.067 & 0.008 & - & - & 0.98 \\
\hline J030011+820238 & 2.415 & 0.097 & 1.379 & 0.046 & 1.078 & 0.102 & 0.586 & 0.034 & 0.265 & 0.008 & 0.177 & 0.022 & - & - & 0.74 \\
\hline J030454+772731 & 0.164 & 0.008 & 0.977 & 0.033 & 0.926 & 0.07 & 0.634 & 0.036 & 0.344 & 0.009 & 0.262 & 0.028 & 0.157 & 0.024 & -0.13 \\
\hline J035150+800437 & 1.534 & 0.061 & 0.546 & 0.018 & 0.368 & 0.033 & 0.211 & 0.012 & 0.078 & 0.004 & 0.041 & 0.005 & - & - & 1.02 \\
\hline J035446+800929 & 1.243 & 0.05 & 0.644 & 0.022 & 0.499 & 0.045 & 0.371 & 0.023 & 0.279 & 0.008 & 0.259 & 0.028 & 0.211 & 0.031 & 0.44 \\
\hline J035629+763742 & 1.969 & 0.079 & 0.629 & 0.021 & 0.397 & 0.036 & 0.252 & 0.016 & 0.127 & 0.007 & 0.098 & 0.011 & - & - & 0.85 \\
\hline J035817+783719 & 1.334 & 0.053 & 0.508 & 0.017 & 0.367 & 0.033 & 0.226 & 0.016 & 0.102 & 0.003 & 0.065 & 0.007 & - & - & 0.85 \\
\hline J040652+763354 & 1.592 & 0.064 & 0.527 & 0.018 & 0.384 & 0.034 & 0.195 & 0.012 & 0.095 & 0.004 & 0.068 & 0.008 & - & - & 0.89 \\
\hline J041045+765645 & 9.406 & 0.376 & 5.62 & 0.189 & 4.538 & 0.336 & 3.512 & 0.197 & 2.215 & 0.054 & 1.769 & 0.182 & 1.279 & 0.142 & 0.47 \\
\hline J041426+761243 & 1.359 & 0.054 & 0.405 & 0.014 & 0.259 & 0.023 & 0.147 & 0.011 & 0.061 & 0.003 & 0.04 & 0.005 & - & - & 1 \\
\hline J041531+842457 & 1.965 & 0.079 & 0.578 & 0.019 & 0.453 & 0.041 & 0.235 & 0.015 & 0.128 & 0.004 & 0.09 & 0.01 & - & - & 0.87 \\
\hline J041946+755915 & 1.491 & 0.06 & 0.427 & 0.014 & 0.273 & 0.025 & 0.166 & 0.01 & 0.076 & 0.004 & 0.045 & 0.005 & - & - & 0.99 \\
\hline J042205+762708 & 3.75 & 0.15 & 1.043 & 0.035 & 0.598 & 0.053 & 0.309 & 0.018 & 0.1 & 0.006 & 0.05 & 0.006 & - & - & 1.22 \\
\hline J042408+765341 & 1.614 & 0.065 & 0.408 & 0.014 & 0.223 & 0.02 & 0.146 & 0.011 & 0.035 & 0.002 & 0.019 & 0.002 & - & - & 1.25 \\
\hline J042918+770911 & 4.702 & 0.188 & 0.945 & 0.032 & 0.517 & 0.044 & 0.266 & 0.015 & 0.085 & 0.004 & 0.026 & 0.003 & - & - & 1.47 \\
\hline J044545+783856 & 1.807 & 0.072 & 0.65 & 0.022 & 0.46 & 0.041 & 0.292 & 0.022 & 0.156 & 0.004 & 0.113 & 0.013 & 0.068 & 0.01 & 0.78 \\
\hline J050731+791257 & 1.859 & 0.074 & 0.629 & 0.021 & 0.496 & 0.044 & 0.307 & 0.019 & 0.155 & 0.004 & 0.122 & 0.014 & 0.066 & 0.01 & 0.77 \\
\hline J050842+843204 & 0.149 & 0.007 & 0.295 & 0.01 & 0.302 & 0.031 & 0.242 & 0.019 & 0.246 & 0.008 & 0.25 & 0.027 & 0.169 & 0.025 & -0.15 \\
\hline J062602+820225 & 0.199 & 0.009 & 0.681 & 0.023 & 0.962 & 0.086 & 0.947 & 0.054 & 0.763 & 0.019 & 0.717 & 0.074 & 0.521 & 0.06 & -0.36 \\
\hline J063012+763245 & 2.187 & 0.088 & 0.783 & 0.026 & 0.7 & 0.063 & 0.339 & 0.025 & 0.152 & 0.009 & 0.092 & 0.011 & - & - & 0.9 \\
\hline J063012+763245 & 1.512 & 0.061 & 0.481 & 0.016 & - & - & 0.193 & 0.015 & 0.085 & 0.004 & 0.053 & 0.006 & - & - & 0.95 \\
\hline J063825+841106 & 3.834 & 0.153 & 1.1 & 0.037 & 0.737 & 0.066 & 0.426 & 0.029 & 0.197 & 0.005 & 0.155 & 0.018 & - & - & 0.91 \\
\hline J064045+781327 & 1.605 & 0.064 & 0.688 & 0.023 & 0.403 & 0.036 & 0.249 & 0.018 & 0.092 & 0.004 & 0.048 & 0.005 & - & - & 0.99 \\
\hline J064558+775502 & 2.054 & 0.082 & 0.608 & 0.02 & 0.458 & 0.041 & - & - & 0.098 & 0.006 & 0.067 & 0.008 & - & - & 0.97 \\
\hline J071452+815153 & 1.704 & 0.068 & 0.551 & 0.018 & 0.419 & 0.037 & 0.255 & 0.016 & 0.141 & 0.004 & 0.127 & 0.014 & - & - & 0.73 \\
\hline J072611+791130 & 0.092 & 0.005 & 0.501 & 0.017 & 0.75 & 0.057 & 0.904 & 0.051 & 0.818 & 0.02 & 0.742 & 0.077 & 0.48 & 0.054 & -0.59 \\
\hline J073433+765813 & 1.361 & 0.055 & 0.475 & 0.016 & 0.282 & 0.025 & 0.217 & 0.013 & 0.098 & 0.006 & 0.074 & 0.009 & - & - & 0.82 \\
\hline J074XXX+802XXX & 9.574 & 0.383 & 3.322 & 0.112 & 2.408 & 0.185 & 1.699 & 0.124 & 0.954 & 0.05 & 0.681 & 0.076 & - & - & 0.75 \\
\hline J075058+824158 & 3.741 & 0.15 & 1.845 & 0.062 & 1.579 & 0.119 & 1.421 & 0.08 & 0.959 & 0.024 & 0.881 & 0.091 & 0.643 & 0.095 & 0.41 \\
\hline J080626+812620 & 1.506 & 0.06 & 0.406 & 0.014 & 0.251 & 0.023 & 0.127 & 0.008 & 0.045 & 0.002 & 0.033 & 0.004 & - & - & 1.08 \\
\hline J080734+784610 & 1.574 & 0.063 & 0.525 & 0.018 & 0.39 & 0.035 & 0.218 & 0.013 & 0.106 & 0.003 & 0.084 & 0.01 & - & - & 0.83 \\
\hline J082550+765313 & 1.903 & 0.076 & 0.689 & 0.023 & 0.491 & 0.044 & 0.303 & 0.018 & 0.159 & 0.004 & 0.121 & 0.014 & 0.028 & 0.004 & 0.78 \\
\hline J083236+800601 & 2.019 & 0.081 & 0.867 & 0.029 & 0.638 & 0.057 & 0.392 & 0.023 & 0.184 & 0.005 & 0.132 & 0.015 & 0.051 & 0.008 & 0.77 \\
\hline J084833+783003 & 5.369 & 0.215 & 1.509 & 0.051 & 0.86 & 0.077 & 0.508 & 0.029 & 0.203 & 0.006 & 0.127 & 0.014 & 0.038 & 0.006 & 1.06 \\
\hline J085834+750121 & 0.239 & 0.01 & 0.948 & 0.032 & 0.578 & 0.052 & 0.302 & 0.017 & 0.097 & 0.006 & 0.048 & 0.005 & - & - & 0.45 \\
\hline J090112+780930 & 1.422 & 0.057 & 0.447 & 0.015 & 0.252 & 0.023 & 0.165 & 0.012 & 0.069 & 0.003 & 0.059 & 0.007 & - & - & 0.9 \\
\hline J090842+834543 & 1.039 & 0.042 & 0.448 & 0.015 & 0.325 & 0.029 & 0.221 & 0.013 & 0.118 & 0.003 & 0.099 & 0.011 & - & - & 0.66 \\
\hline J092016+862845 & 2.122 & 0.085 & 0.519 & 0.017 & 0.345 & 0.031 & 0.173 & 0.013 & 0.065 & 0.003 & 0.036 & 0.004 & - & - & 1.15 \\
\hline J093239+790629 & 9.234 & 0.369 & 2.241 & 0.075 & 1.333 & 0.119 & 0.667 & 0.038 & 0.235 & 0.006 & 0.127 & 0.014 & - & - & 1.21 \\
\hline J093817+781528 & 2.062 & 0.083 & 0.699 & 0.023 & 0.426 & 0.038 & 0.272 & 0.016 & 0.125 & 0.004 & 0.075 & 0.009 & - & - & 0.94 \\
\hline J093923+831526 & 11.592 & 0.464 & 2.953 & 0.099 & 1.802 & 0.134 & 0.917 & 0.052 & 0.304 & 0.009 & 0.171 & 0.02 & - & - & 1.19 \\
\hline J094440+825408 & 1.904 & 0.076 & 0.735 & 0.025 & 0.54 & 0.048 & 0.358 & 0.023 & 0.173 & 0.006 & 0.129 & 0.015 & - & - & 0.76 \\
\hline J095559+791134 & 1.295 & 0.052 & 0.415 & 0.014 & 0.254 & 0.023 & 0.152 & 0.009 & 0.061 & 0.003 & 0.047 & 0.005 & - & - & 0.94 \\
\hline $\mathrm{J} 100005+812702$ & 2.541 & 0.102 & 0.864 & 0.029 & 0.561 & 0.05 & 0.304 & 0.017 & 0.122 & 0.007 & 0.069 & 0.008 & - & - & 1.02 \\
\hline $\mathrm{J} 100741+813150$ & 3.632 & 0.145 & 0.855 & 0.029 & 0.496 & 0.044 & 0.257 & 0.015 & 0.096 & 0.003 & 0.05 & 0.006 & - & - & 1.21 \\
\hline $\mathrm{J} 100949+810719$ & 1.5 & 0.06 & 0.455 & 0.015 & 0.328 & 0.029 & 0.195 & 0.012 & 0.081 & 0.004 & 0.062 & 0.007 & - & - & 0.9 \\
\hline $\mathrm{J} 101015+825014$ & 0.741 & 0.03 & 0.504 & 0.017 & 0.507 & 0.045 & 0.622 & 0.035 & 0.607 & 0.015 & 0.628 & 0.066 & 0.548 & 0.064 & 0.05 \\
\hline $\mathrm{J} 101037+765052$ & 3.076 & 0.123 & 0.806 & 0.027 & 0.522 & 0.047 & 0.235 & 0.02 & 0.071 & 0.004 & 0.033 & 0.004 & - & - & 1.28 \\
\hline $\mathrm{J} 101 \mathrm{XXX}+855 \mathrm{XXX}$ & 1.973 & 0.079 & 0.81 & 0.027 & 0.537 & 0.045 & 0.303 & 0.034 & 0.109 & 0.007 & 0.066 & 0.008 & - & - & 0.96 \\
\hline $\mathrm{J} 101734+810517$ & 2.894 & 0.116 & 1.172 & 0.039 & 0.838 & 0.075 & 0.512 & 0.03 & 0.194 & 0.006 & 0.12 & 0.014 & - & - & 0.9 \\
\hline $\mathrm{J} 102326+803255$ & 6.905 & 0.276 & 1.807 & 0.061 & 1.144 & 0.102 & 0.627 & 0.036 & 0.185 & 0.005 & 0.093 & 0.011 & - & - & 1.22 \\
\hline $\mathrm{J} 102926+785241$ & 2.559 & 0.102 & 1.111 & 0.037 & 0.741 & 0.066 & 0.525 & 0.03 & 0.25 & 0.006 & 0.164 & 0.019 & - & - & 0.78 \\
\hline $\mathrm{J} 104423+805439$ & 0.643 & 0.026 & 0.828 & 0.028 & 0.829 & 0.074 & 0.961 & 0.054 & 1.212 & 0.029 & 1.362 & 0.14 & 1.206 & 0.134 & -0.21 \\
\hline
\end{tabular}


Table 4. continued

\begin{tabular}{|c|c|c|c|c|c|c|c|c|c|c|c|c|c|c|c|}
\hline 1 & 2 & 3 & 4 & 5 & 6 & 7 & 8 & 9 & 10 & 11 & 12 & 13 & 14 & 15 & 16 \\
\hline $\mathrm{J} 105150+791341$ & 1.894 & 0.076 & 0.525 & 0.018 & 0.314 & 0.028 & 0.179 & 0.01 & 0.078 & 0.004 & 0.06 & 0.007 & - & - & 0.98 \\
\hline $\mathrm{J} 110405+793253$ & 0.338 & 0.014 & 0.514 & 0.017 & 0.491 & 0.044 & 0.377 & 0.021 & 0.237 & 0.006 & 0.172 & 0.018 & 0.11 & 0.016 & 0.19 \\
\hline $\mathrm{J} 110412+765859$ & 7.554 & 0.302 & 2.341 & 0.079 & 1.518 & 0.113 & 0.887 & 0.05 & 0.395 & 0.01 & 0.224 & 0.023 & - & - & 0.99 \\
\hline $\mathrm{J} 111342+765449$ & 1.534 & 0.061 & 0.471 & 0.016 & 0.29 & 0.026 & 0.183 & 0.011 & 0.095 & 0.005 & 0.066 & 0.007 & - & - & 0.89 \\
\hline $\mathrm{J} 112342+773123$ & 1.516 & 0.061 & 0.407 & 0.014 & 0.228 & 0.02 & 0.151 & 0.009 & 0.055 & 0.003 & 0.036 & 0.004 & - & - & 1.06 \\
\hline $\mathrm{J} 114829+782721$ & 0.97 & 0.039 & 0.42 & 0.014 & 0.3 & 0.027 & 0.206 & 0.012 & 0.088 & 0.005 & 0.074 & 0.009 & - & - & 0.73 \\
\hline $\mathrm{J} 115312+805829$ & 1.39 & 0.056 & 1.343 & 0.045 & 1.887 & 0.146 & 1.922 & 0.108 & 1.715 & 0.042 & 1.696 & 0.175 & 1.251 & 0.138 & -0.06 \\
\hline $\mathrm{J} 115504+753439$ & 1.822 & 0.073 & 0.817 & 0.027 & 0.594 & 0.053 & 0.407 & 0.025 & 0.201 & 0.006 & 0.137 & 0.016 & - & - & 0.73 \\
\hline J115522+815709 & 2.017 & 0.081 & 0.671 & 0.023 & 0.428 & 0.038 & 0.227 & 0.019 & 0.1 & 0.004 & 0.065 & 0.007 & - & - & 0.97 \\
\hline $\mathrm{J} 115608+823505$ & 1.4 & 0.056 & 0.404 & 0.014 & 0.25 & 0.023 & 0.175 & 0.02 & 0.065 & 0.003 & 0.056 & 0.007 & - & - & 0.91 \\
\hline $\mathrm{J} 115713+811824$ & 1.269 & 0.051 & 0.981 & 0.033 & 0.766 & 0.06 & 0.556 & 0.033 & 0.301 & 0.009 & 0.222 & 0.023 & - & - & 0.49 \\
\hline $\mathrm{J} 122015+792732$ & 1.409 & 0.056 & 0.517 & 0.017 & 0.334 & 0.03 & 0.214 & 0.012 & 0.084 & 0.004 & 0.043 & 0.005 & - & - & 0.99 \\
\hline $\mathrm{J} 122340+804004$ & 1.204 & 0.048 & 0.705 & 0.024 & 0.701 & 0.063 & 0.752 & 0.043 & 0.767 & 0.019 & 0.808 & 0.083 & 0.775 & 0.087 & 0.11 \\
\hline $\mathrm{J} 122518+860839$ & 1.532 & 0.061 & 0.453 & 0.015 & 0.294 & 0.026 & 0.164 & 0.016 & 0.082 & 0.004 & 0.041 & 0.005 & - & - & 1.02 \\
\hline $\mathrm{J} 123708+835704$ & 1.909 & 0.076 & 0.778 & 0.026 & 0.667 & 0.051 & 0.333 & 0.02 & 0.186 & 0.005 & 0.122 & 0.014 & - & - & 0.78 \\
\hline $\mathrm{J} 125736+834231$ & 1.145 & 0.046 & 0.475 & 0.016 & 0.375 & 0.034 & 0.27 & 0.019 & 0.176 & 0.005 & 0.164 & 0.019 & - & - & 0.55 \\
\hline $\mathrm{J} 130035+805438$ & 4.837 & 0.194 & 1.251 & 0.042 & 0.777 & 0.069 & 0.386 & 0.022 & 0.166 & 0.004 & 0.107 & 0.012 & - & - & 1.08 \\
\hline $\mathrm{J} 130538+815626$ & 1.734 & 0.069 & 0.49 & 0.016 & 0.32 & 0.029 & 0.17 & 0.01 & 0.077 & 0.004 & 0.054 & 0.006 & - & - & 0.98 \\
\hline $\mathrm{J} 130609+800825$ & 2.218 & 0.089 & 0.785 & 0.026 & 0.597 & 0.053 & 0.365 & 0.022 & 0.18 & 0.007 & 0.142 & 0.016 & - & - & 0.78 \\
\hline $\mathrm{J} 130705+764918$ & 1.178 & 0.047 & 0.754 & 0.025 & 0.531 & 0.048 & 0.359 & 0.021 & 0.18 & 0.006 & 0.124 & 0.014 & - & - & 0.64 \\
\hline $\mathrm{J} 130811+854424$ & 1.711 & 0.069 & 0.593 & 0.02 & 0.418 & 0.037 & 0.236 & 0.015 & 0.156 & 0.009 & 0.108 & 0.012 & - & - & 0.78 \\
\hline $\mathrm{J} 131723+821916$ & 3.126 & 0.125 & 0.869 & 0.029 & 0.46 & 0.041 & 0.29 & 0.017 & 0.114 & 0.007 & 0.08 & 0.009 & - & - & 1.04 \\
\hline $\mathrm{J} 132053+845011$ & 0.702 & 0.028 & 0.436 & 0.015 & 0.484 & 0.044 & 0.28 & 0.021 & 0.211 & 0.007 & 0.189 & 0.022 & - & - & 0.37 \\
\hline $\mathrm{J} 132145+831613$ & 0.829 & 0.033 & 0.565 & 0.019 & 0.485 & 0.043 & 0.333 & 0.025 & 0.275 & 0.015 & 0.262 & 0.03 & - & - & 0.33 \\
\hline $\mathrm{J} 132331+780947$ & 1.192 & 0.048 & 0.482 & 0.016 & 0.346 & 0.031 & 0.23 & 0.014 & 0.106 & 0.003 & 0.072 & 0.008 & - & - & 0.79 \\
\hline $\mathrm{J} 132351+794251$ & 0.574 & 0.023 & 0.599 & 0.02 & 0.642 & 0.057 & 0.575 & 0.033 & 0.476 & 0.012 & 0.435 & 0.046 & 0.331 & 0.042 & 0.08 \\
\hline $\mathrm{J} 135639+794340$ & 2.121 & 0.085 & 0.579 & 0.019 & 0.349 & 0.031 & 0.189 & 0.011 & 0.072 & 0.004 & 0.036 & 0.004 & - & - & 1.15 \\
\hline $\mathrm{J} 135755+764320$ & 0.419 & 0.017 & 0.647 & 0.022 & 0.575 & 0.051 & 0.7 & 0.04 & 0.769 & 0.02 & 0.819 & 0.085 & 0.761 & 0.085 & -0.19 \\
\hline $\mathrm{J} 141419+790547$ & 1.445 & 0.058 & 0.424 & 0.014 & 0.251 & 0.023 & 0.153 & 0.01 & 0.082 & 0.004 & 0.056 & 0.007 & - & - & 0.92 \\
\hline $\mathrm{J} 141718+805939$ & 1.519 & 0.061 & 0.541 & 0.018 & 0.356 & 0.032 & 0.217 & 0.016 & 0.115 & 0.005 & 0.077 & 0.009 & - & - & 0.84 \\
\hline $\mathrm{J} 141947+760033$ & 3.216 & 0.129 & 0.981 & 0.033 & 0.635 & 0.057 & 0.388 & 0.022 & 0.192 & 0.007 & 0.137 & 0.016 & 0.058 & 0.009 & 0.89 \\
\hline $\mathrm{J} 142248+770416$ & 1.094 & 0.044 & 0.541 & 0.018 & 0.329 & 0.029 & 0.173 & 0.01 & 0.063 & 0.003 & 0.029 & 0.003 & - & - & 1.03 \\
\hline $\mathrm{J} 142613+794607$ & 1.257 & 0.05 & 0.407 & 0.014 & 0.266 & 0.024 & 0.168 & 0.012 & 0.067 & 0.003 & 0.036 & 0.004 & - & - & 1 \\
\hline $\mathrm{J} 143547+760526$ & 2.644 & 0.106 & 1.304 & 0.044 & 0.979 & 0.087 & 0.724 & 0.041 & 0.428 & 0.011 & 0.323 & 0.034 & 0.191 & 0.028 & 0.59 \\
\hline $\mathrm{J} 144314+770726$ & 6.648 & 0.266 & 1.882 & 0.063 & 1.069 & 0.095 & 0.552 & 0.034 & 0.225 & 0.006 & 0.131 & 0.015 & - & - & 1.11 \\
\hline $\mathrm{J} 144709+765621$ & 6.168 & 0.247 & 1.667 & 0.056 & 0.997 & 0.089 & 0.559 & 0.032 & 0.226 & 0.006 & 0.144 & 0.016 & - & - & 1.06 \\
\hline $\mathrm{J} 150008+751851$ & 3.858 & 0.154 & 0.784 & 0.026 & 0.395 & 0.035 & 0.19 & 0.011 & 0.076 & 0.004 & 0.044 & 0.005 & - & - & 1.26 \\
\hline $\mathrm{J} 150207+860811$ & 0.863 & 0.035 & 0.416 & 0.014 & 0.379 & 0.034 & 0.229 & 0.014 & 0.108 & 0.003 & 0.087 & 0.01 & - & - & 0.65 \\
\hline $\mathrm{J} 151304+814326$ & 2.144 & 0.086 & 0.782 & 0.026 & 0.554 & 0.05 & 0.302 & 0.022 & 0.134 & 0.004 & 0.088 & 0.01 & - & - & 0.9 \\
\hline $\mathrm{J} 153112+770604$ & 1.446 & 0.058 & 0.566 & 0.019 & 0.346 & 0.031 & 0.18 & 0.01 & 0.056 & 0.003 & 0.021 & 0.002 & - & - & 1.2 \\
\hline $\mathrm{J} 153700+815431$ & 0.2 & 0.009 & 0.433 & 0.015 & 0.39 & 0.035 & 0.314 & 0.018 & 0.2 & 0.005 & 0.163 & 0.019 & 0.095 & 0.014 & 0.06 \\
\hline $\mathrm{J} 160222+801558$ & 4.507 & 0.18 & 1.016 & 0.034 & 0.515 & 0.046 & 0.255 & 0.015 & - & - & 0.049 & 0.005 & - & - & 1.28 \\
\hline $\mathrm{J} 160929+793954$ & 2.806 & 0.112 & 1.239 & 0.042 & 0.965 & 0.086 & 0.711 & 0.04 & 0.431 & 0.012 & 0.333 & 0.035 & 0.193 & 0.028 & 0.6 \\
\hline $\mathrm{J} 161940+854921$ & 3.965 & 0.159 & 1.643 & 0.055 & 1.322 & 0.098 & 0.801 & 0.046 & 0.433 & 0.011 & 0.319 & 0.034 & - & - & 0.71 \\
\hline $\mathrm{J} 163051+823345$ & - & - & 0.875 & 0.029 & 0.378 & 0.034 & 0.259 & 0.019 & 0.086 & 0.005 & 0.052 & 0.006 & - & - & 1.36 \\
\hline $\mathrm{J} 163226+823220$ & - & - & 0.802 & 0.027 & 0.777 & 0.064 & 0.644 & 0.037 & 0.555 & 0.014 & 0.615 & 0.065 & 0.647 & 0.096 & 0.13 \\
\hline $\mathrm{J} 163925+863153$ & 2.234 & 0.089 & 0.852 & 0.029 & 0.633 & 0.057 & 0.418 & 0.026 & 0.233 & 0.006 & 0.173 & 0.019 & - & - & 0.72 \\
\hline $\mathrm{J} 164843+754628$ & 5.799 & 0.232 & 1.942 & 0.065 & 1.246 & 0.111 & 0.746 & 0.042 & 0.365 & 0.01 & 0.245 & 0.026 & 0.109 & 0.016 & 0.89 \\
\hline $\mathrm{J} 165752+792808$ & 2.458 & 0.098 & 0.872 & 0.029 & 0.599 & 0.054 & 0.399 & 0.024 & 0.179 & 0.006 & 0.123 & 0.014 & - & - & 0.85 \\
\hline $\mathrm{J} 171416+761245$ & 1.916 & 0.077 & 0.459 & 0.015 & 0.268 & 0.024 & 0.139 & 0.008 & 0.056 & 0.003 & 0.031 & 0.004 & - & - & 1.17 \\
\hline $\mathrm{J} 172359+765312$ & 0.216 & 0.009 & 0.424 & 0.014 & 0.342 & 0.03 & 0.358 & 0.02 & 0.343 & 0.011 & 0.315 & 0.033 & 0.279 & 0.042 & -0.11 \\
\hline $\mathrm{J} 172529+770805$ & 0.979 & 0.039 & 0.565 & 0.019 & 0.425 & 0.038 & 0.262 & 0.016 & 0.134 & 0.005 & 0.096 & 0.011 & - & - & 0.66 \\
\hline J172550+772624 & 3.077 & 0.123 & 1.163 & 0.039 & 0.795 & 0.071 & 0.499 & 0.028 & 0.27 & 0.007 & 0.206 & 0.023 & 0.132 & 0.019 & 0.76 \\
\hline $\mathrm{J} 173021+794916$ & 4.444 & 0.178 & 1.02 & 0.034 & 0.648 & 0.058 & 0.309 & 0.033 & 0.126 & 0.004 & 0.064 & 0.007 & - & - & 1.2 \\
\hline $\mathrm{J} 173734+844543$ & 1.971 & 0.079 & 0.444 & 0.015 & 0.258 & 0.023 & 0.128 & 0.009 & 0.054 & 0.003 & 0.029 & 0.003 & - & - & 1.19 \\
\hline $\mathrm{J} 175056+814736$ & 1.139 & 0.046 & 0.44 & 0.015 & 0.269 & 0.024 & 0.161 & 0.011 & 0.054 & 0.003 & 0.033 & 0.004 & - & - & 1 \\
\hline $\mathrm{J} 180045+782804$ & 1.918 & 0.077 & 2.224 & 0.075 & 2.679 & 0.205 & 2.883 & 0.162 & 2.674 & 0.067 & 2.69 & 0.278 & 2.427 & 0.272 & -0.1 \\
\hline $\mathrm{J} 183712+851449$ & 1.852 & 0.074 & 0.69 & 0.023 & 0.526 & 0.047 & 0.246 & 0.016 & 0.113 & 0.004 & 0.057 & 0.007 & - & - & 0.98 \\
\hline $\mathrm{J} 184 \mathrm{XXX}+794 \mathrm{XXX}$ & 30.187 & 1.207 & 12.944 & 0.435 & 9.44 & 0.859 & 5.94 & 0.416 & 3.63 & 0.37 & - & - & - & - & 0.85 \\
\hline $\mathrm{J} 184502+765230$ & 1.517 & 0.061 & 0.536 & 0.018 & 0.347 & 0.031 & 0.251 & 0.016 & 0.101 & 0.006 & 0.061 & 0.007 & - & - & 0.91 \\
\hline $\mathrm{J} 185750+774636$ & 1.636 & 0.066 & 0.474 & 0.016 & 0.291 & 0.026 & 0.166 & 0.01 & 0.074 & 0.004 & 0.042 & 0.005 & - & - & 1.03 \\
\hline $\mathrm{J} 190350+853647$ & 2.533 & 0.101 & 0.905 & 0.03 & 0.63 & 0.056 & 0.411 & 0.03 & 0.192 & 0.005 & 0.147 & 0.017 & - & - & 0.8 \\
\hline $\mathrm{J} 190919+781330$ & 0.823 & 0.033 & 0.465 & 0.016 & 0.33 & 0.03 & 0.202 & 0.012 & 0.094 & 0.003 & 0.057 & 0.007 & - & - & 0.75 \\
\hline $\mathrm{J} 193419+795606$ & 2.721 & 0.109 & 0.765 & 0.026 & 0.518 & 0.046 & 0.231 & 0.016 & 0.101 & 0.006 & 0.049 & 0.005 & - & - & 1.13 \\
\hline $\mathrm{J} 193739+835629$ & 0.844 & 0.034 & 0.43 & 0.014 & 0.392 & 0.035 & 0.239 & 0.015 & 0.22 & 0.008 & 0.265 & 0.028 & - & - & 0.33 \\
\hline $\mathrm{J} 194136+850138$ & 2.477 & 0.099 & 0.662 & 0.022 & 0.596 & 0.053 & 0.29 & 0.02 & 0.129 & 0.007 & 0.094 & 0.011 & - & - & 0.92 \\
\hline $\mathrm{J} 194340+785829$ & 1.107 & 0.044 & 0.499 & 0.017 & 0.444 & 0.04 & 0.205 & 0.014 & 0.111 & 0.004 & 0.078 & 0.009 & - & - & 0.75 \\
\hline $\mathrm{J} 194420+781602$ & 1.631 & 0.065 & 0.503 & 0.017 & 0.322 & 0.029 & 0.147 & 0.009 & 0.044 & 0.002 & - & - & - & - & 1.45 \\
\hline $\mathrm{J} 194958+765413$ & 1.827 & 0.073 & 0.51 & 0.017 & 0.312 & 0.028 & 0.164 & 0.009 & 0.074 & 0.004 & 0.051 & 0.006 & - & - & 1.01 \\
\hline $\mathrm{J} 200531+775243$ & 0.806 & 0.032 & 1.061 & 0.036 & 1.343 & 0.102 & 1.463 & 0.083 & 1.453 & 0.035 & 1.428 & 0.147 & 1.324 & 0.146 & -0.16 \\
\hline $\mathrm{J} 202235+761126$ & 0.567 & 0.023 & 0.429 & 0.014 & 0.5 & 0.045 & 0.449 & 0.025 & 0.414 & 0.01 & 0.393 & 0.041 & 0.369 & 0.055 & 0.1 \\
\hline $\mathrm{J} 2042 \mathrm{XX}+750 \mathrm{XXX}$ & - & - & 1.144 & 0.038 & 1.172 & 0.1 & 0.842 & 0.062 & 0.555 & 0.028 & - & - & - & - & 0.71 \\
\hline
\end{tabular}


Table 4. continued

\begin{tabular}{|c|c|c|c|c|c|c|c|c|c|c|c|c|c|c|c|}
\hline 1 & 2 & 3 & 4 & 5 & 6 & 7 & 8 & 9 & 10 & 11 & 12 & 13 & 14 & 15 & 16 \\
\hline $\mathrm{J} 204541+762510$ & 3.238 & 0.13 & 0.953 & 0.032 & 0.603 & 0.054 & 0.474 & 0.027 & 0.257 & 0.008 & 0.201 & 0.023 & - & - & 0.79 \\
\hline $\mathrm{J} 205033+752622$ & 2.107 & 0.084 & 0.556 & 0.019 & 0.365 & 0.032 & 0.182 & 0.012 & 0.082 & 0.004 & 0.046 & 0.005 & - & - & 1.08 \\
\hline $\mathrm{J} 210407+763307$ & 15.52 & 0.621 & 3.891 & 0.131 & 2.27 & 0.169 & 1.314 & 0.074 & 0.486 & 0.012 & 0.259 & 0.027 & - & - & 1.16 \\
\hline $\mathrm{J} 2118 \mathrm{XX}+751 \mathrm{XXX}$ & 4.717 & 0.189 & 1.261 & 0.042 & 0.66 & 0.059 & 0.419 & 0.027 & 0.135 & 0.004 & 0.078 & 0.009 & - & - & 1.16 \\
\hline $\mathrm{J} 211956+765734$ & 1.188 & 0.048 & 0.432 & 0.015 & 0.307 & 0.027 & 0.15 & 0.011 & 0.082 & 0.004 & 0.05 & 0.006 & - & - & 0.89 \\
\hline $\mathrm{J} 212926+845326$ & 3.514 & 0.141 & 1.274 & 0.043 & 0.809 & 0.072 & 0.482 & 0.035 & 0.168 & 0.007 & 0.072 & 0.008 & - & - & 1.1 \\
\hline $\mathrm{J} 213008+835730$ & 5.098 & 0.204 & 1.798 & 0.06 & 1.332 & 0.119 & 0.846 & 0.048 & 0.382 & 0.01 & 0.279 & 0.032 & - & - & 0.82 \\
\hline $\mathrm{J} 213139+843011$ & 0.445 & 0.018 & 0.677 & 0.023 & 0.647 & 0.058 & 0.422 & 0.032 & 0.241 & 0.007 & 0.185 & 0.021 & - & - & 0.25 \\
\hline $\mathrm{J} 213334+823905$ & 1.93 & 0.077 & 0.915 & 0.031 & 0.809 & 0.072 & 0.617 & 0.035 & 0.433 & 0.01 & 0.39 & 0.045 & 0.192 & 0.028 & 0.45 \\
\hline $\mathrm{J} 213929+833953$ & 1.418 & 0.057 & 0.48 & 0.016 & 0.306 & 0.027 & 0.217 & 0.012 & 0.107 & 0.006 & 0.081 & 0.009 & - & - & 0.81 \\
\hline $\mathrm{J} 214928+754045$ & 1.735 & 0.069 & 0.524 & 0.018 & 0.329 & 0.029 & 0.202 & 0.012 & 0.104 & 0.006 & 0.073 & 0.009 & - & - & 0.9 \\
\hline $\mathrm{J} 215657+833714$ & 0.474 & 0.019 & 0.474 & 0.016 & 0.442 & 0.039 & 0.285 & 0.022 & 0.214 & 0.009 & 0.184 & 0.021 & - & - & 0.27 \\
\hline $\mathrm{J} 215712+764642$ & 2.283 & 0.091 & 0.777 & 0.026 & 0.56 & 0.05 & 0.309 & 0.018 & 0.149 & 0.006 & 0.091 & 0.011 & - & - & 0.91 \\
\hline $\mathrm{J} 220955+835356$ & 1.787 & 0.072 & 0.578 & 0.019 & 0.4 & 0.036 & 0.191 & 0.018 & 0.09 & 0.005 & 0.069 & 0.008 & - & - & 0.92 \\
\hline $\mathrm{J} 222800+753219$ & 2.039 & 0.082 & 0.651 & 0.022 & 0.429 & 0.038 & 0.289 & 0.018 & 0.142 & 0.005 & 0.111 & 0.013 & - & - & 0.82 \\
\hline $\mathrm{J} 224714+855542$ & 1.432 & 0.057 & 0.516 & 0.017 & 0.356 & 0.032 & 0.205 & 0.013 & 0.098 & 0.006 & 0.076 & 0.009 & - & - & 0.83 \\
\hline $\mathrm{J} 230122+795406$ & 1.447 & 0.058 & 0.431 & 0.014 & 0.291 & 0.026 & 0.154 & 0.01 & 0.077 & 0.004 & 0.044 & 0.005 & - & - & 0.99 \\
\hline $\mathrm{J} 230138+820015$ & 1.518 & 0.061 & 0.45 & 0.015 & 0.378 & 0.034 & 0.196 & 0.016 & 0.085 & 0.004 & 0.046 & 0.005 & - & - & 0.99 \\
\hline $\mathrm{J} 232503+791715$ & 0.705 & 0.028 & 1.136 & 0.038 & 0.912 & 0.082 & 0.578 & 0.037 & 0.285 & 0.007 & 0.189 & 0.021 & - & - & 0.37 \\
\hline $\mathrm{J} 232640+823158$ & 2.964 & 0.119 & 1.001 & 0.034 & 0.728 & 0.065 & 0.35 & 0.022 & 0.16 & 0.009 & 0.066 & 0.007 & - & - & 1.07 \\
\hline $\mathrm{J} 232803+761738$ & 1.466 & 0.059 & 0.459 & 0.015 & 0.26 & 0.023 & 0.172 & 0.01 & 0.072 & 0.004 & 0.053 & 0.006 & - & - & 0.94 \\
\hline $\mathrm{J} 234403+822640$ & 5.667 & 0.227 & 3.777 & 0.127 & 2.904 & 0.259 & 1.787 & 0.102 & 0.896 & 0.022 & 0.65 & 0.067 & 0.292 & 0.043 & 0.61 \\
\hline $\mathrm{J} 234914+751744$ & 1.428 & 0.057 & 0.43 & 0.014 & 0.322 & 0.029 & 0.2 & 0.013 & 0.117 & 0.007 & 0.096 & 0.011 & - & - & 0.76 \\
\hline $\mathrm{J} 235413+804753$ & 1.634 & 0.065 & 0.482 & 0.016 & 0.307 & 0.027 & 0.178 & 0.01 & 0.075 & 0.004 & 0.058 & 0.007 & - & - & 0.94 \\
\hline $\mathrm{J} 2355 \mathrm{XX}+795 \mathrm{XXX}$ & 6.463 & 0.259 & 1.706 & 0.057 & 0.967 & 0.073 & 0.603 & 0.034 & 0.196 & 0.007 & 0.094 & 0.011 & - & - & 1.2 \\
\hline $\mathrm{J} 235622+815252$ & 0.569 & 0.023 & 0.521 & 0.017 & 0.454 & 0.041 & 0.5 & 0.032 & 0.586 & 0.016 & 0.667 & 0.071 & 0.713 & 0.08 & -0.04 \\
\hline
\end{tabular}

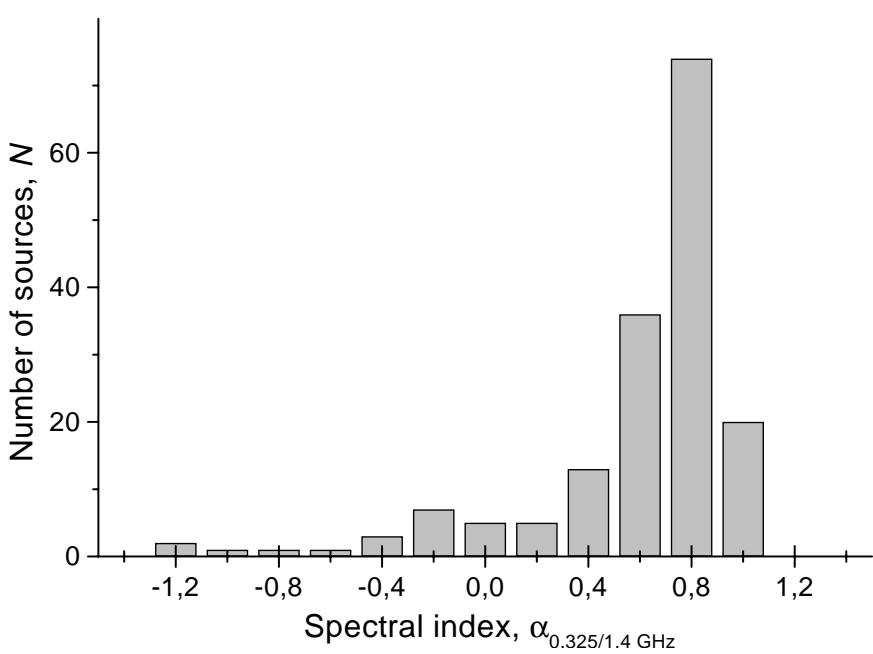

Fig. 3. Spectral index distribution between 0.325 and $1.4 \mathrm{GHz}$

whole frequency range measured; of these, 4 show weak GPS behavior (see below) and 3 show weakly rising spectra up to the highest frequency observed and may also be GPS sources. 4 sources show evidence for a flat spectrum component at the higher frequencies of the survey.

There is a potential 10 percent of this survey which are GPS sources. These are believed to be compact objects with a peak in their spectra at $\mathrm{GHz}$ frequencies in the redshift frame of emission. They are characterized by a difference of spectral index ("curvature") on either side of the peak of more than 0.6 (de Vries et al. 1997). The observed peak frequency may be as low as $0.5 \mathrm{GHz}$ (Marecki et al. 1999). There are 9 sources in our list which satisfy these spectral characteristics. It is possible that several may be giant radio galaxies with low frequency absorption (for

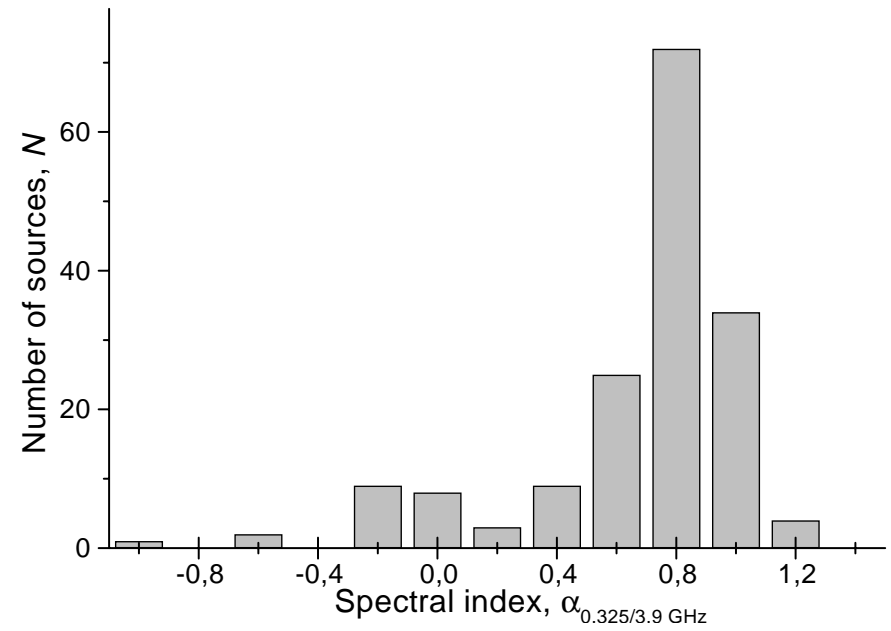

Fig. 4. Spectral index distribution between 0.325 and $3.9 \mathrm{GHz}$

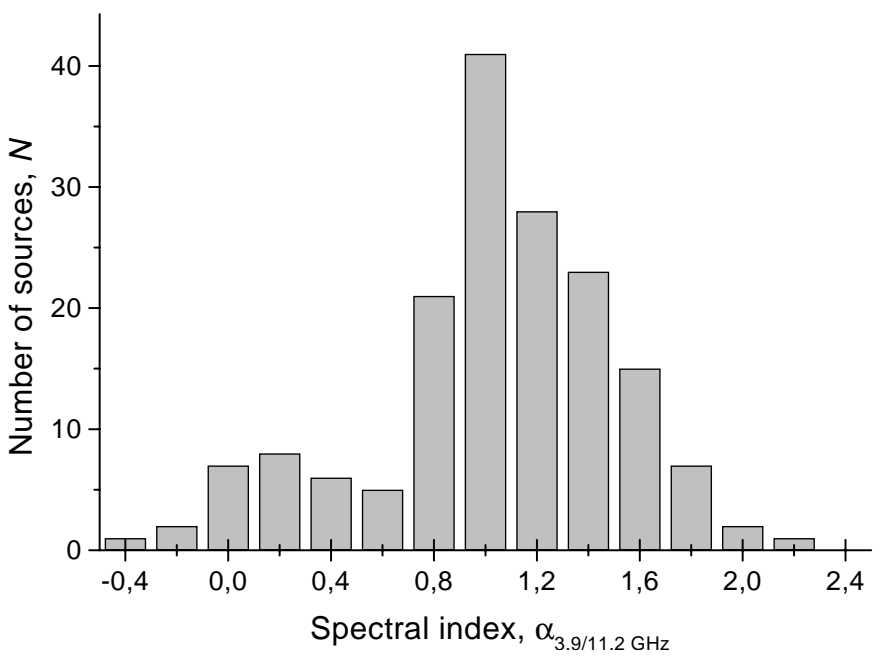

Fig. 5. Spectral index distribution between 3.9 and $11.2 \mathrm{GHz}$ 


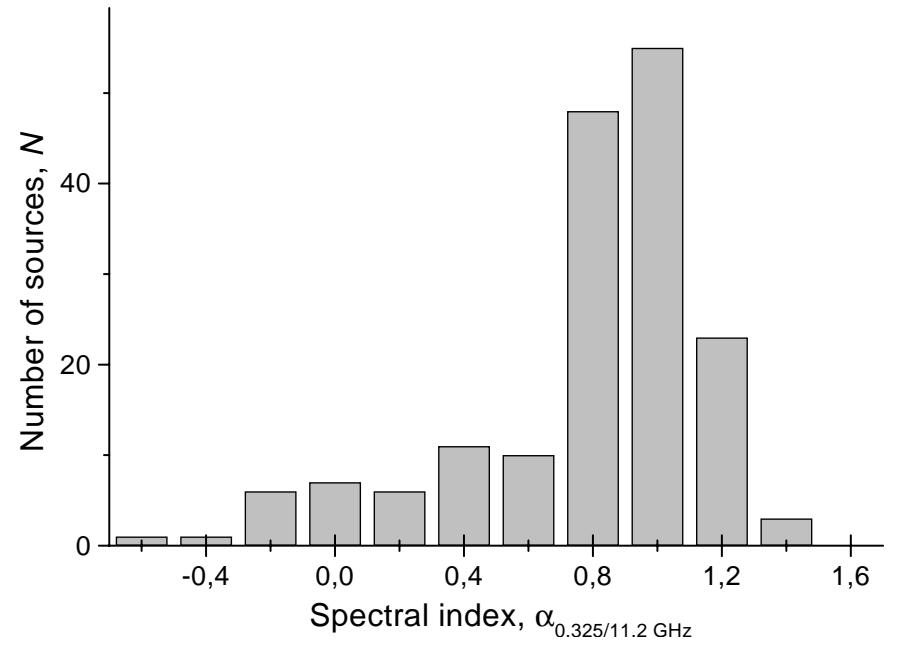

Fig. 6. Spectral index distribution between 0.325 and $11.2 \mathrm{GHz}$

example $085834+750121)$; mapping will be required to establish their compactness. Four sources $(132351+794251$; $172359+765312 ; 180045+782804 ; 200531+775243)$ have a curvature of 0.4 to 0.6 , just below the canonical limit of de Vries et al.; they are compact as indicated by their spectra and are potential GPS sources. Three sources $(104423+805439 ; 135755+764320 ; 235622+815252)$ have spectra which are weakly rising with $\alpha=0.2$ to 0.3 at the highest frequencies of observation. These are also likely GPS sources with peak frequencies $\geq 10$ to $20 \mathrm{GHz}$.

Acknowledgements. This research is partially supported by the Russian Foundation for Basis Research Project No. 9802-16428 and Russian Federal Program "Astronomy" Project No. 1.2.5.1. VS acknowledges the receipt of NATO/Royal Society Postdoctoral Fellowship.

The authors used extensively the database CATS (http://cats.sao.ru, Verkhodanov et al. 1997) of the Special Astrophysical Observatory (Russia) in the search for counterparts in the radio catalogues.

\section{References}

Aliakberov, K., Mingaliev, M., Naugol'naya, M., et al. 1985, Astrophys. Issled. (Izvestiya SAO), 19, 60

Berlin, A., \& Friedman, P. 1996, Real Time Radiometric Data Processing Against Electromagnetic Pollution, in 25th General Assembly of the International Union of Radio Science abstracts, Lille, France, 750

Berlin, A., Maksyasheva, A., Nizhelskij, N., et al. 1997, Spread spectrum radiometers of RATAN-600 radio telescope, in The problems of Modern Radio Astronomy, 27th Radio Astronomy Conference, Sankt-Peterburg, 115

Condon, J. J., Cotton, W. D., Greisen, E. W., et al. 1998, AJ, 115,1693

Giardino, G., Asareh, H., Melhuish, S. J., et al. 2000, MNRAS, 313,689

Gregory, P. C., Scott, W. K., Douglas, K., \& Condon, J. J. 1996, ApJS, 103, 427

Korolkov, D. V, \& Pariiskii, Yu. N. 1979, Sky Telesc., 57, 324

Kovalev, Y. Y, Nizhelsky, N. A., Kovalev, Yu. A., et al. 1999, A\&AS, 139, 545

Kuehr, H., Witzel, A., Pauliny-Toth, I. I. K., \& Nauber, U. 1981, A\&AS, 45, 367

Marecki, A., Falcke, H., Niezgoda, J., Garrington, S. T., \& Patnaik, A. R. 1999, A\&AS, 135, 273

Melhuish, S. J., Davies, R. D., Mingaliev, M. G., \& Stolyarov, V. A. 2001, in preparation

Mingaliev, M., Botashev, A., \& Stolyarov, V. 1998, Multifrequency monitoring of a sample of extragalactic radio sources, In IAU Colloquium 164: Radio Emission from Galactic and Extragalactic Compact Sources, ed. J. A. Zensus, G. B. Taylor, \& J. M. Wrobel, ASP Conf. Ser., 144,279

Parijskij, Yu. 1993, IEEE Antennas Propagation Magazine (IAPM), 35, 7

Pauliny-Toth, I. I. K., Witzel, A., Preuss, E., Baldwin, J. E., \& Hills, R. E. 1978, A\&AS, 34, 253

Perley, R., \& Taylor, G. 1999, VLA Calibrator Manual,

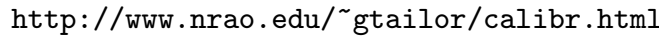

Rengelink, R. B., Tang, Y., de Bruyn, A. G., et al. 1997, A\&AS, 124,259

Trushkin, S. 1985, Ph.D. Thesis, Special Astrophysical Observatory of Russian Academy of Sciences

Verkhodanov, O., Trushkin, S., Andernach, H., \& Chernenkov, V. 1997, The CATS database to operate with astrophysical catalogs, in Astronomical Data Analysis Software and Systems VI, ed. G. Hunt, \& H. E. Payne, ASP Conf. Ser, 125,322

Verkhodanov, O. 1997, Multiwaves continuum data reduction at RATAN-600, in Astronomical Data Analysis Software and Systems VI, ed. G. Hunt, \& H. E. Payne, ASP Conf. Ser., 125, 46

de Vries, W. H., Barthel, P. D., \& O’Dea, C. P. 1997, A\&A, 321,105 